\title{
A SIMPLE R-BANDING TECHNIQUE BY BrdU- HOECHST TREATMENT AND GIEMSA STAINING FOLLOWING HEATING AND ULTRAVIOLET EXPOSURE
}

\author{
Atsushi Ieshima, Taeko Yorita, and Kenzo Takeshita \\ Division of Child Neurology, Tottori University School of Medicine, \\ 86 Nishimachi, Yonago 683, Japan
}

\begin{abstract}
Summary A simple R-banding technique was presented. Peripheral leukocytes were incorporated with 5'-bromodeoxy uridine for 6 to $8 \mathrm{hr}$ prior to havesting. R-banded metaphases were obtained by Giemsa staining following 33258 Hoechst staining for $15 \mathrm{~min}$ and heating at $60^{\circ} \mathrm{C}$ for $80 \mathrm{~min}$ in $2 \times \mathrm{SSC}$ with exposure to ultraviolet. This method has many advantages; 1) the possibility of obtaining a permanent R-banding preparation immediately after harvest, 2 ) feasible demonstration of the latereplicating $\mathrm{X}$ chromosome, and 3 ) no requirement of a fluorescence microscope.
\end{abstract}

\section{INTRODUCTION}

R-banding by the heat-denaturation method of Dutrillaux and Lejeune (1971) and several modifications of this method (Eiberg, 1975; Verma and Lubs, 1975) have been widely applied. The method of 5'-bromodeoxy uridine (BrdU) pretreatment in culture (RBA method) has been commonly used for the studying the replication of the $\mathrm{X}$ chromosome, and for demonstration of the $\mathrm{R}$ band with a high reproducibility (Dutrillaux, 1975; Pai and Thomas, 1980). The principal limitations of the RBA method are that a fluorescence microscope is required, and that slides should be processed for R-banding within 6-9 days after the harvest. Pai and Thomas (1980) pointed out the possibility of an R-banding technique with HoechstGiemsa staining. Camargo and Cervenka (1982) demonstrated an R-banding technique using cell synchronization combined with BrdU pulsing and fluorochrome-photolysis-Giemsa (FPG) differential staining. This method was essentially developed for the demonstration of sister chromatid exchange in the metaphase chromosome (Perry and Wolff, 1974; Korenberg and Freedlender, 1974). But this technique is too complicated for routine use.

We report here a simple R-banding technique using the BrdU-Hoechst 33258Giemsa method. Permanent R-banded preparations are obtained immediately by our method. 


\section{MATERIALS AND METHODS}

Whole blood samples from 60 consecutive patients referred to us for cytogenetic diagnosis were cultured for $72 \mathrm{hr}$ at $37^{\circ} \mathrm{C}$ in Dulbecco's modified Eagle's medium with $0.1 \%$ glucose and $0.37 \% \mathrm{NaHCO}_{3}$ supplemented with $10 \%$ fetal bovine serum, 2.5\% phytohemagglutinin (Gibco, $\mathrm{M}$ form) and anti-biotics. BrdU was added to a final concentration of $50 \mu \mathrm{g} / \mathrm{ml}$ during the last 6 to $8 \mathrm{hr}$ of culture. Corcemid (Gibco, $0.03 \mu \mathrm{g} / \mathrm{ml}$ ) and ethidium bromide (Sigma, $3 \mu \mathrm{g} / \mathrm{ml}$ ) were added to the culture $90 \mathrm{~min}$ prior to fixation. Chromosome preparations were obtained by the ignition technique following hypotonic treatment with $3: 10.075 \mathrm{M} \mathrm{KCl}$ : $1 \%$ sodium citrate for $15 \mathrm{~min}$ at $37^{\circ} \mathrm{C}$, and fixation with a $3: 1$ methanol : acetic acid fixative. The slides were immediately stained in Hoechst 33258 (Wako Pure Chemicals; $50 \mu \mathrm{g} / \mathrm{ml}$ distilled water) for $15 \mathrm{~min}$ and then rinsed briefly in deionized water. The preparations were mounted in $2 \times \mathrm{SSC}(0.3 \mathrm{M}$ sodium chloride $-0.03 \mathrm{M}$ trisodium citrate) and the coverslips ringed with nail enamel to prevent evaporation. The slides were placed on a hot plate (surface temperature of approximately $60^{\circ} \mathrm{C}$ ) and exposed to a ultraviolet (UV) light (distance of about $17 \mathrm{~cm}$ ) for about $80 \mathrm{~min}$. The coverslips were then removed and the slides rinsed in running water. The slides were then stained with phosphate-buffered Giemsa $(3 \%, \mathrm{pH} 6.8)$ for $20 \mathrm{~min}$.

\section{RESULTS AND DISCUSSION}

Figure 1 shows an R-banded karyotype of a patient with $48, \mathrm{XXXY}$ after 33258 Hoechst and Giemsa staining. The Giemsa preparation is permanent, and can be easily studied with a compound microscope. The majority of cells on any given slide showed the typical R-banding pattern. This technique provided excellentquality karyotypes of the R-band in all patients studied. Occasionally differences of the staining intensity between euchromatic regions of homologues were observed. But they were negligible when more than two metaphases were analyzed. When the R-banding pattern was not obtained at the first attempt, it was necessary to alter the duration of UV exposure and heating time to 60-90 min. Since a betterquality karyotype of the R-band was observed in less contracted chromosomes, ethidium bromide was added during the last $90 \mathrm{~min}$ according to the method of Ikeuchi and Sasaki (1979) with slight modifications. R-banding was feasible within three weeks of slide storage at room temperature. The period of BrdU pretreatment was changed from 3 to $12 \mathrm{hr}$. On 3 to $4 \mathrm{hr}$ treatment, R-banding patterns were faintly visible and nearly all of the metaphases darkly stained. With the longest treatment (more than $10 \mathrm{hr}$ ), weakly stained metaphases were observed in many cells. In the 6-8 hr treatment group, R-banding of the best quality was observed. This phenomenon was also observed for the RBA method and discussed by Dutrillaux et al. (1976) and Camargo and Cervenka (1982).

The late-replicating $\mathrm{X}$ chromosomes were shown as an unbanded or variably 


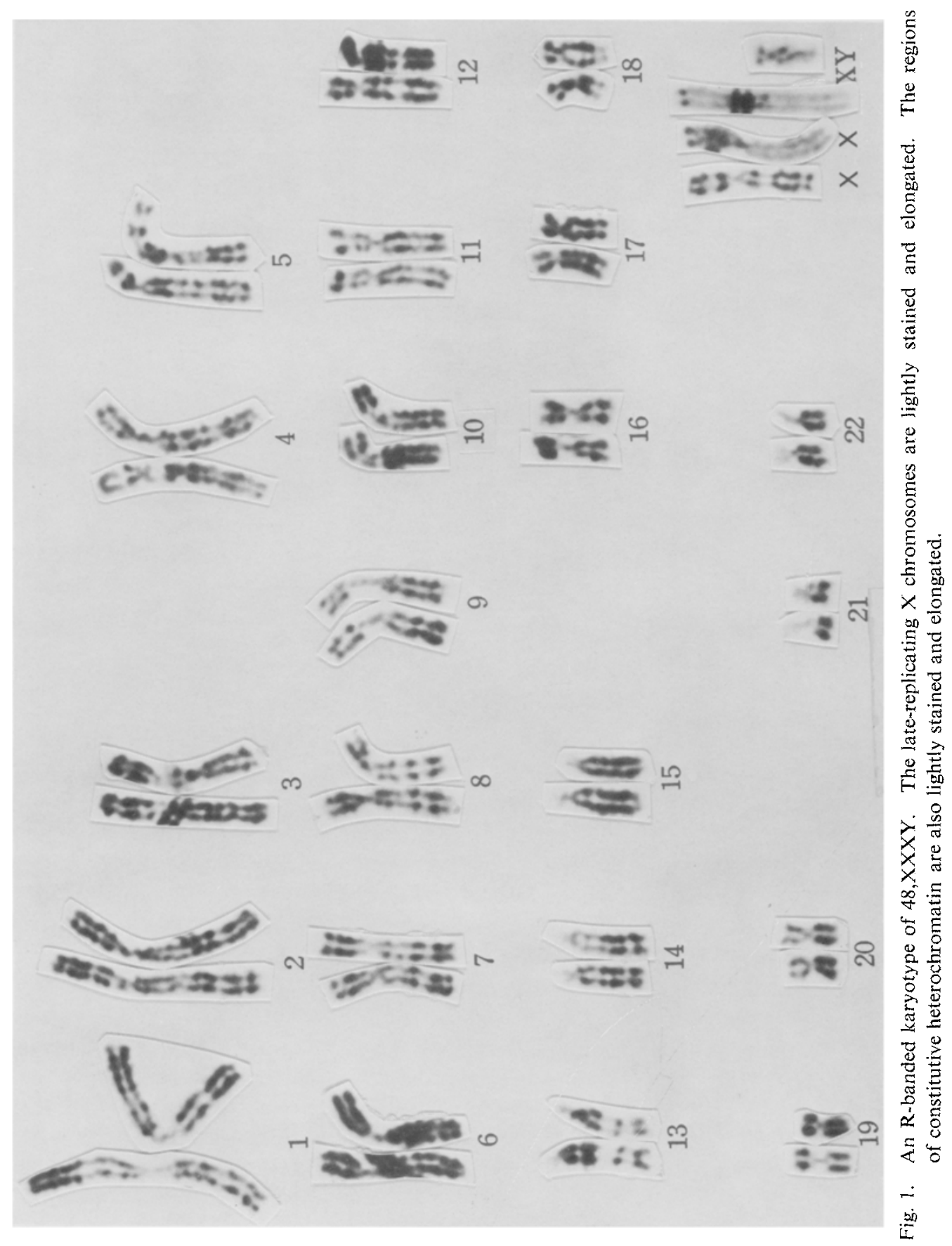

Vol. 29, No. 2, 1984 


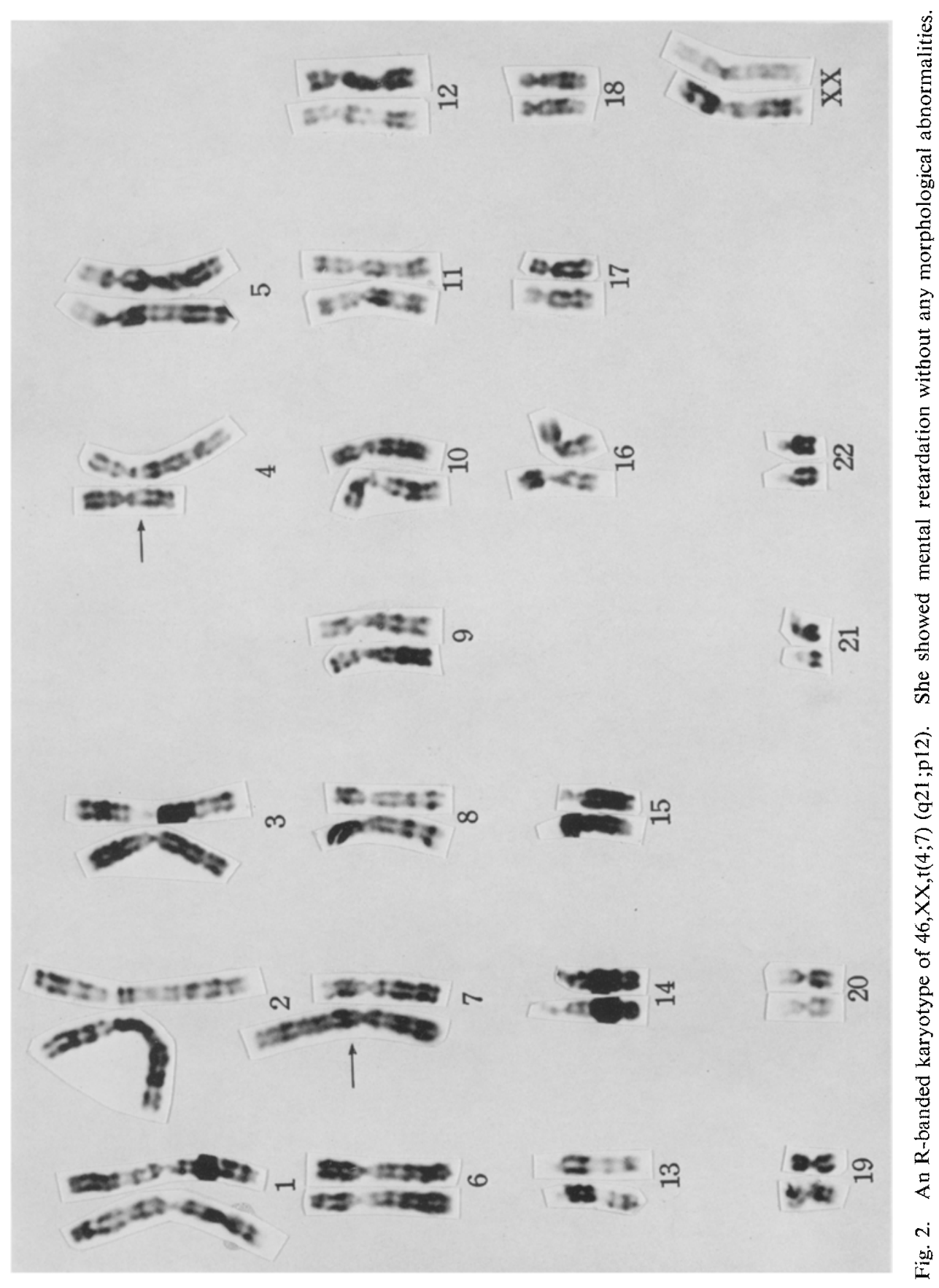




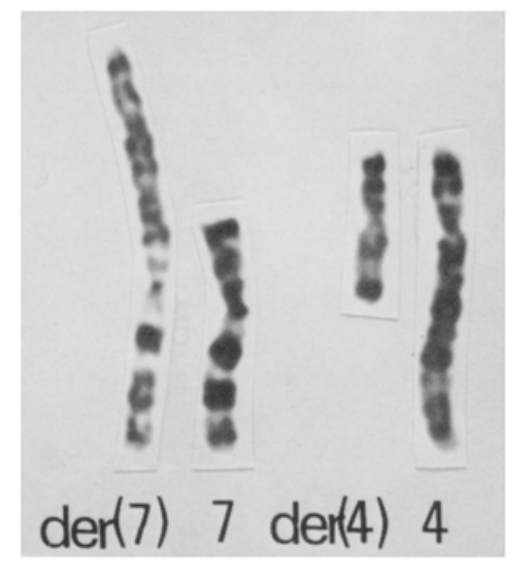

Fig. 3. A partial karyotype of $46, \mathrm{XX}, \mathrm{t}(4 ; 7)(\mathrm{q} 21 ; \mathrm{p} 12)$ by $\mathrm{G}$ banding.

banded structure. Although it is necessary to count and analyze a large number of cells in a patient with mosaic Turner syndrome, mosaicism can be easily analyzed by this method. A late-replicating study of the $\mathrm{X}$ chromosome can also be performed easily. The pitfall of this method is that the structural abnormalities of the $X$ chromosome may be misjudged.

Figure 2 shows an R-banded karyotype of $46, \mathrm{XX}, \mathrm{t}(4 ; 7)(\mathrm{q} 21 ; \mathrm{pl} 2)$. Figure 3 shows a partial karyotype. The combination with $R$ and $G$ band is believed to be the most informative. Since more than ten slides can be processed for Rbanding at the same time, this method can save much time, effort and money, and can be adapted for routine use.

The advantages of this method are 1) the feasibility of getting permanent $\mathrm{R}$ banding preparations $0-3$ weeks after harvesting, 2) no necessity of a fluorescence microscope, and 3 ) the ability to demonstrate the late-replicating $X$ chromosome. Our technique is simple and highly reproducible and may be of value for practical application.

Acknowledgement We are grateful to Dr. Y. Kuroki, Director of Div. of Medical Genetics, Kanagawa Children's Medical Center, for his kind advice and encouragement.

\section{REFERENCES}

Camargo, M. and Cervenka, J. 1982. Patterns of DNA replication of human chromosome. II. Replication map and replication model. Am. J. Hum. Genet. 34: 757-780

Dutrillaux, B. and Lejeune, J. 1971. Sur une novelle technique d'analyse du caryotype human. CR. Acad. Sci. [D] (Paris) 272: 2638-2640

Dutrillaux, B. 1975. Traitment discontinues par le BrdU et coloration par l'acridine orange: Obtention de marquages R, Q et intermédiares. Chromosoma 52: 261-273

Dutrillaux, B., Couturier, J., Richer, C.L., and Viegas-Péquignot, E. 1976. Sequence of DNA 
replication in $277 \mathrm{R}$ - and Q-bands of human chromosomes using a BrdU treatment. Chromosoma 58: 51-61

Eiberg, H.G. 1975. G, R and C band patterns of human chromosomes produced by heat treatment in organic and inorganic salt solutions. Clin. Genet. 4: 556-562

Ikeuchi, T. and Sasaki, M. 1979. Accumulation of early mitotic cells in ethidium bromide-treated human lymphocyte cultures. Proc. Jpn. Acad. 55(B): 15-18.

Korenberg, J.R. and Freedlender, E.F., 1974. Giemsa technique for the detection of sister chromatid exchanges. Chromosoma 48: $355-360$

Pai, G.S. and Thomas, C.H., 1980. A new R-banding technique in clinical cytogenetics. Hum. Genet. 54: 41-45

Perry, P. and Wolff, S., 1974. New Giemsa method for the differential staining of sister chromatids. Nature (London) 251: 156-158

Verma, R.S. and Lubs, H.A. 1975. A simple R banding technique. Am. J. Hum. Genet. 27: 110117 